\title{
INTERMEDIATE BAND TO CONDUCTION BAND OPTICAL ABSORPTION IN ZnTe:O
}

\author{
E. Antolín ${ }^{1,2}$, C. Chen ${ }^{3}$, I. Ramiro ${ }^{1}$, J. Foley ${ }^{3}$, E. López ${ }^{1}$, I. Artacho ${ }^{1}$, J. Hwang ${ }^{3}$, A. Teran ${ }^{3}$, E. Hernández ${ }^{1}$, \\ C. Tablero ${ }^{1}$, A. Martí ${ }^{1}$, J.D. Phillips ${ }^{3}$ and A. Luque ${ }^{1}$ \\ ${ }^{1}$ Instituto de Energía Solar, Universidad Politécnica de Madrid, Spain \\ ${ }^{2}$ Instituto de Microelectrónica de Madrid, CSIC, Madrid, Spain. \\ ${ }^{3}$ Department of Electrical Engineering and Computer Science, The University of Michigan, Ann Arbor MI, USA
}

\begin{abstract}
ZnTe doped with high concentrations of oxygen has been proposed in previous works as intermediate band (IB) material for photovoltaic applications. The existence of extra optical transitions related to the presence of an IB has already been demonstrated in this material and it has been possible to measure the absorption coefficient of the transitions from the valence band (VB) to the IB. In this work we present the first measurement of the absorption coefficient associated to transitions from the IB to the conduction band (CB) in $\mathrm{ZnTe}$ :O.
\end{abstract}

The samples used are $4 \mu \mathrm{m}$ thick ZnTe layers with or without $O$ in a concentration $\sim 10^{19} \mathrm{~cm}^{-3}$, which have been grown on semi-insulating GaAs substrates by molecular beam epitaxy (MBE). The IB-CB absorption coefficient peaks for photon energies $\sim 0.4 \mathrm{eV}$. It is extracted from reflectance and transmittance spectra measured using Fourier Transform Infrared (FTIR) spectroscopy. Under typical FTIR measurement conditions (low light intensity, broadband spectrum) the absorption coefficient in IB-toCB transitions reaches $700 \mathrm{~cm}^{-1}$. This is much weaker than the one observed for VB-IB absorption. This result is consistent with the fact that the IB is expected to be nearly empty of electrons under equilibrium conditions in ZnTe(:O).

The absorption for VB to IB transitions is also observed in the same samples through reflectance measurements performed in the visible range using a monochromator. These measurements are compared with the quantum efficiency (QE) from solar cells fabricated under similar conditions.

\section{THE SUB-BANDGAP ABSORPTION IN THE IBSC}

The intermediate band solar cell (IBSC) [1] is a photovoltaic device designed to achieve a high efficiency through the exploitation of sub-bandgap photons. In this device, low energy photons are absorbed thanks to the existence of an extra band, the IB, within the semiconductor band gap of the absorber. As it is shown in Figure 1 this band enables two different transitions: $V B \rightarrow I B$ (labeled "1") and IB $\rightarrow$ CB (labeled "2"). For the device to achieve high efficiency, it is of the utmost importance that both transitions are operative, and not just one of them (if we exclude impact ionization processes). In this respect, it is important that the IB is disconnected from the external contacts by the layers called "emitters". Since only electrons and holes exit the cell, and not IB carriers, it is not possible to extract photocurrent if only one subbandgap transition is used. In practice it may be possible to extract a current if the lattice thermal energy is used to sustain a carrier escape flux that replaces the failing optical transition. But in that case, the price paid is a reduction of the voltage. By disconnecting the IB from the contacts and exploiting simultaneously the two subbandgap transitions, the IBSC can work at a voltage similar to the one it would have if no IB was present.

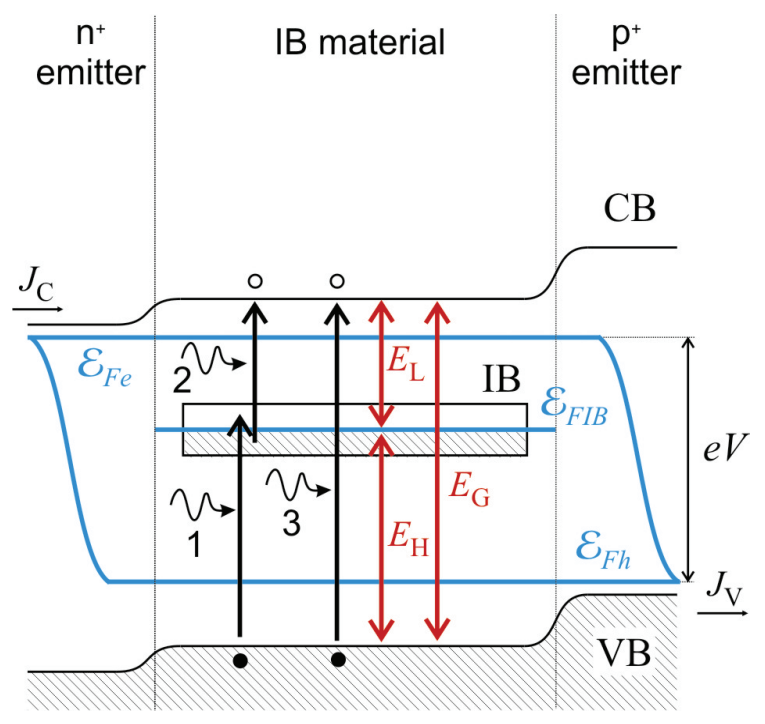

Figure 1 Simplified band diagram of an IBSC illustrating its operation. The IB divides the semiconductor gap in two sub-bandgaps $\left(E_{\mathrm{L}}\right.$ and $\left.E_{\mathrm{H}}\right)$ and enables the absorption of extra photons (labeled 1 and 2).

In order to generate a significant sub-bandgap photocurrent it is important that the IB is partially filled, so that both transitions can take place at a high rate [2]. There are two ways of achieving this. The first one is to have the Fermi level located at the IB under equilibrium conditions. It has been predicted that this occurs naturally for some IB materials [3], but in other cases it is also possible to tune the occupancy of the IB through codoping [4]. If the density of states in the IB is sufficiently high, the quasi-Fermi level associated to it will remain at the IB under illumination conditions. This situation of prefilling of the IB is generally assumed for efficiency limit 
calculations. The second possibility is to relay on photofilling, i.e., the optical absorption in one transition provides the carriers to be pumped by the other transition. It has been demonstrated theoretically that the efficiency that can be achieved relying on photo-filling only approaches the pre-filling efficiency under high illumination. In [5] it is shown that the difference between the two cases becomes small for a concentration of 1000 suns (assuming equal optical cross-section in both sub-bandgap transitions).

Another important requirement for optimum performance of the IBSC is the selectivity of absorption coefficients. The efficiency of the device would decrease if part of the energy of the photons is lost because they are absorbed in a transition of energy lower than possible. For instance, if we consider an IB material as depicted in Figure 1 where the IB is closer to the $C B$, then it would be a loss that photons with energy enough to be absorbed in a VB-IB transition are absorbed in an IB-CB transition. Ideally, the way to avoid this is that the absorption coefficients $\alpha_{\mathrm{Vc}}, \alpha_{\mathrm{VI}}$ and $\alpha_{I C}$, associated respectively to the VB-CB, VB-IB and IB-CB transitions are selective. This implies that $\alpha_{I C}$ should be zero in the range where $\alpha_{\mathrm{VI}}$ is non-zero (photon energies $\geq E_{\mathrm{H}}$ ) and $\alpha_{\mathrm{VI}}$ should behave analogously with respect to $\alpha_{v c}$

\section{ZnTe:O AS IB MATERIAL}

One way that is currently being investigated to engineer an IB material is the doping of a semiconductor with an impurity species that introduces levels within the gap. It has been proposed that, if the impurity concentration is very high, the levels become delocalized, which suppresses the non-radiative recombination processes that usually accompany the presence of levels in the gap [6]. Among the possible candidates, ZnTe doped with oxygen has been one of the most studied materials [7-11] (in some cases adding a small amount of $\mathrm{Mn}$ to enlarge the total bandgap). Fabrication of IBSC prototypes has been reported $[8,10]$ and sub-bandgap transitions have been identified in $\mathrm{ZnTe}: \mathrm{O}$ by modulated photoreflectance techniques $[7,10]$. Also, it has been possible to measure the absorption coefficient of the VB to IB transitions, which has been reported to be high $\left(\sim 10^{4} \mathrm{~cm}^{-1}\right)$ by different authors [10,11].

\section{EXPERIMENTAL DESCRIPTION}

In this work, we have grown by MBE relatively thick samples $(4 \mu \mathrm{m})$ of $\mathrm{ZnTe}(: \mathrm{O})$ on $\mathrm{GaAs}(001)$ substrates (see Figure 2, right) and have used them to measure the absorption coefficient associated to IB-CB transitions. The MBE system includes solid source effusion cells for $\mathrm{Zn}$ and $\mathrm{Te}$, and a RF plasma source for oxygen incorporation (and nitrogen when required as $p$ dopant). An oxygen flow rate of 1 SCCM was applied (1 cubic centimeter per minute at standard pressure and temperature), which results in an oxygen partial pressure during growth in the order of $10^{-5}$ Torr. Under these conditions the $O$ content is estimated to be in the order of $10^{19} \mathrm{~cm}^{-3}$, assuming similar incorporation rates to $\mathrm{N}$. The band diagram of the material depends on the amount of $O$ that is introduced $[7,12,13]$. Under these conditions, based on previous PL studies [8], we expect a band diagram as depicted in Figure 2, left. As a reference, a similar ZnTe sample with no oxygen has also been grown. The GaAs substrates used in all cases are semi-insulating to avoid free carrier absorption in the IR.

The objectives of this work are: (1) to demonstrate that both sub-bandgap absorptions take place in $\mathrm{ZnTe:O}$, and (2) to initiate the empirical study of the aspects discussed in the introduction on a fabricated IB material (impact of the IB occupancy on the absorption and selectivity of the absorption coefficients). It is known the $\mathrm{ZnTe}$ is naturally $p$-doped due to the presence of $\mathrm{Zn}$ vacancies. Since $\mathrm{O}$ is an isovalent impurity, there is no reason for the $O$ doping to change the p-character of $\mathrm{ZnTe}$ if the $\mathrm{O}$ is Tesubstitutional. Prior capacitance-voltage measurements based on materials grown under similar conditions have indicated a background p-type carrier concentration $<10^{16}$ $\mathrm{cm}^{-3}$ for $\mathrm{ZnTeO}$. Therefore, the IB is most likely to be almost empty under equilibrium.

To measure the absorption of the VB-IB transition, a setup has been used including a monochromator covering the visible range, Si detector and lock-in amplifier. For those energies, the GaAs substrate absorbs the light and only reflectance spectra can be obtained (diagram on the left, Figure 3). For the measurement of the IB-CB transition, a FTIR spectrometer has been used. In this case it is possible to obtain reflectance and transmittance spectra (diagram on the right, Figure 3).

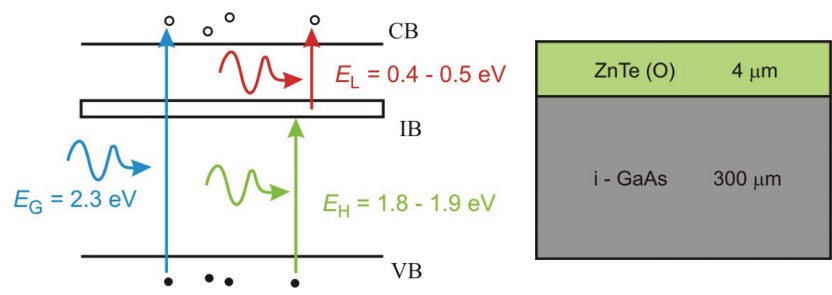

Figure 2: Left, expected band diagram of the ZnTe:O bulk IB material. Right, structure of the test samples grown for this absorption study.

\section{RESULTS}

Figure 4 shows the reflectance spectra measured in the visible range on a $\mathrm{ZnTe}$ and a $\mathrm{ZnTe}: \mathrm{O}$ sample $\left(30^{\circ}\right.$ with respect to normal incidence) using the monochromator set up. For ZnTe (upper plot) it can be seen a flat reflectance for energies above the fundamental bandgap and an interference oscillation for energies below it. This is the typical measurement of a semiconductor film around its bandgap. The flat component that is present for any photon energy is the surface reflectance $\left(R_{1}\right.$ in Figure 3 , left) and the interfering components $\left(R_{2} \ldots R_{\mathrm{n}}\right)$ appear only for energies at which the layer is transparent. 
In the case of ZnTe:O (lower plot) the interference is strongly attenuated for sub-bandgap energies, indicating VB-IB absorption in the layer. Since the absorption is complete in a large part of the range, it is not possible to extract from this measurement an accurate absorption coefficient value. It can be estimated, given the thickness of the layer, that between 2 and $2.3 \mathrm{eV}$ the VB-IB absorption coefficient has to be at least in the order of $10^{4}$ $\mathrm{cm}^{-1}$, which is in agreement with previous reports [10].
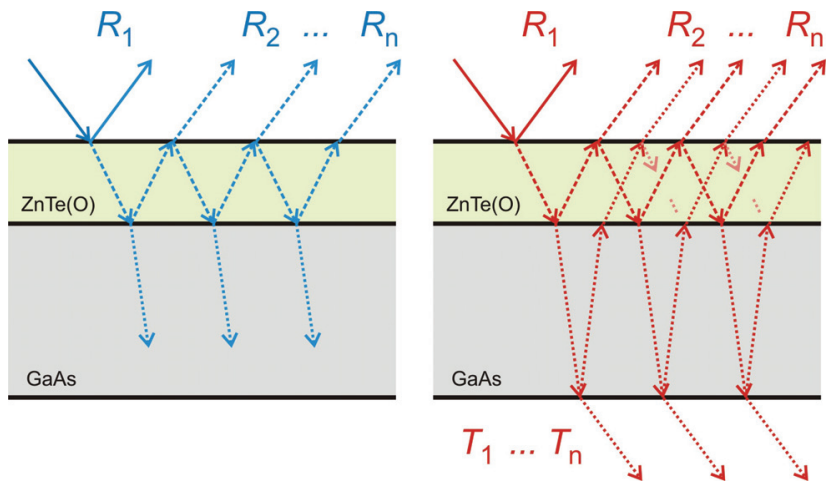

Figure 3: Light beam components present in the reflectance and transmittance experiments. On the left, photons have energy larger than the gap of GaAs. On the right, photon energy is below the gap of GaAs.

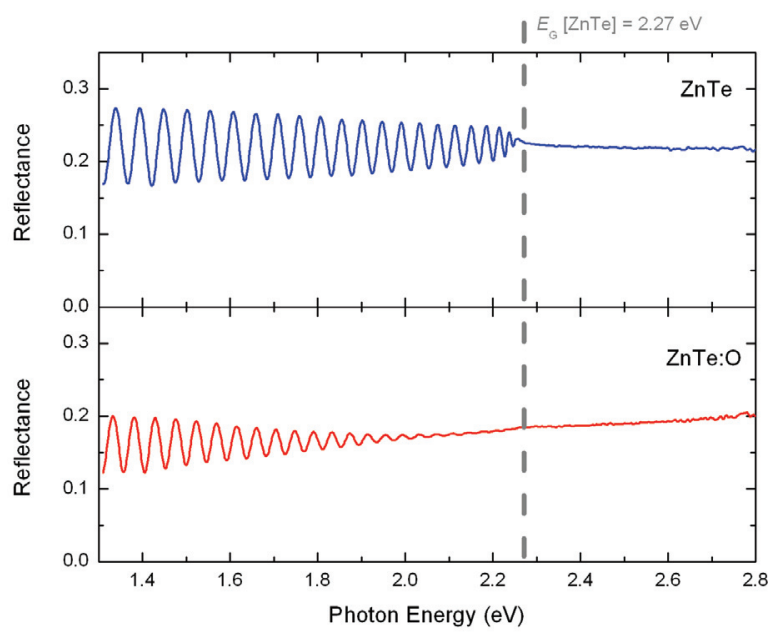

Figure 4. Measurement of the reflectance of $\mathrm{ZnTe}$ and ZnTe:O samples.

Figure 5 shows the reflectance $(R)$ and transmittance $(T)$ spectra under normal incidence measured in the IR range on the same samples using a FTIR. It can be seen a dip around $0.4 \mathrm{eV}$ in the transmittance of the $\mathrm{ZnTe}: \mathrm{O}$ sample which is not present for the ZnTe sample. We interpret that feature as the effect of the absorption in the IB-CB transition. From those measurements we can obtain the absorbance, $A$, of the film as $A=1-T-R$. Then, we can extract the absorption coefficient, $\alpha$, from the expression $A=1-\exp (-\alpha d)$, where $d$ is the thickness of the film.
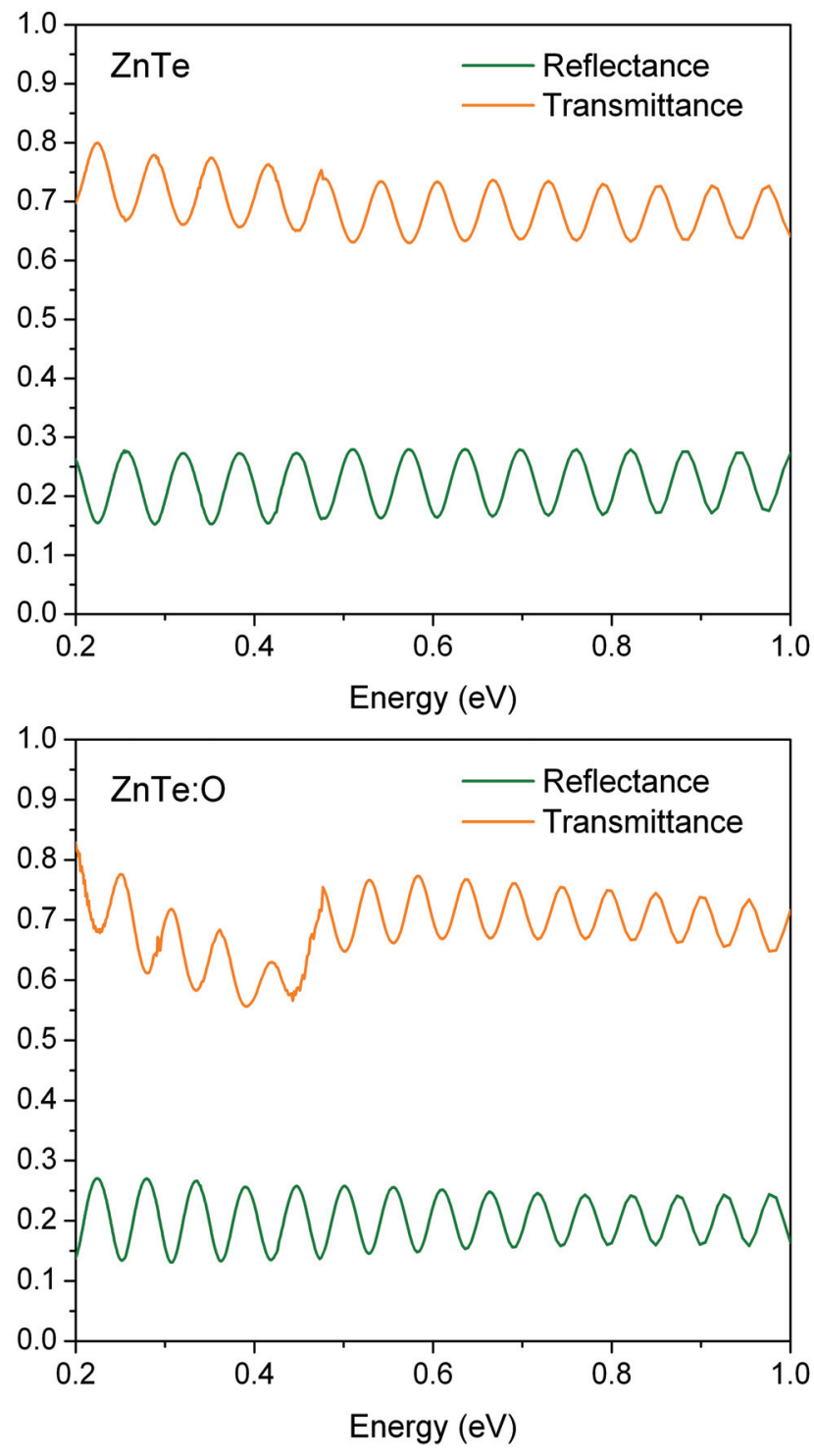

Figure 5: Reflectance and transmittance spectra in the IR measured with FTIR on the ZnTe (top) and ZnTe:O (bottom) samples (same samples as in Figure 4).

We have estimated a thickness of $3.5 \mu \mathrm{m}$ for the $\mathrm{ZnTe}$ film and $4.0 \mu \mathrm{m}$ for the $\mathrm{ZnTe}: \mathrm{O}$ film using the well-known fact that in the interference pattern $T$ reaches a maximum and $R$ a minimum when $2 n d \approx m \lambda$ ( $n$ is the refractive index, $\lambda$ the wavelength and $m$ an integer). Here we have assumed for $\mathrm{ZnTeO}$ the same $n$ as for $\mathrm{ZnTe}$.

The resulting absorption coefficients are shown in Figure 6. Since the absorption peak observed at 0.3-0.4 eV for $\mathrm{ZnTe}: \mathrm{O}$ is clearly above the amplitude of the interference 
oscillations, this measurement constitutes a good approximation to the real bulk absorption coefficient of the material. If the absorption peak was of the same order than the amplitude of the interference oscillations, modeling would be required to distinguish the real absorption coefficient from the $\alpha$ that we have defined above. It is also remarkabable that this absorption has been detectable at room temperature. The absorption baseline measured for $\mathrm{ZnTe}$ determines the level of inaccuracy introduced by the non-ideal surfaces of the grown samples (scattering).

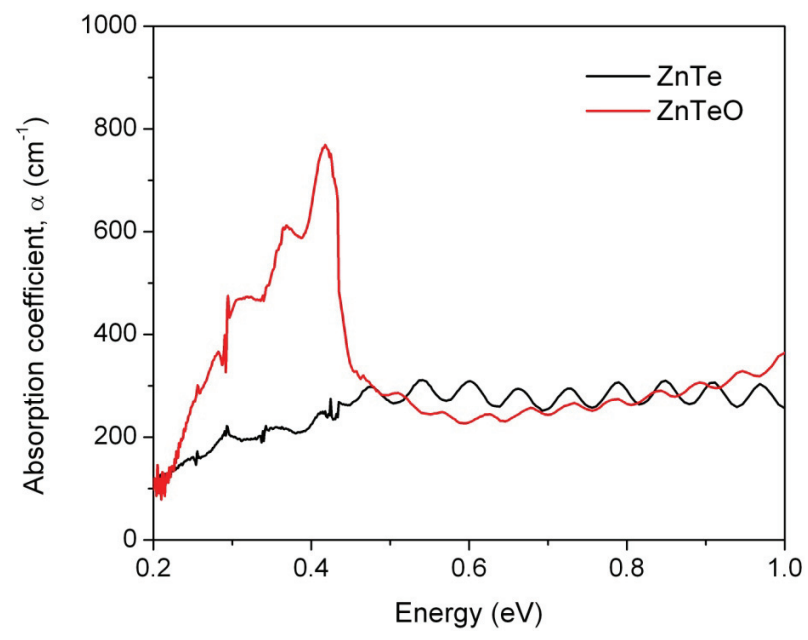

Figure 6. Absorption coefficient associated to the $I B \rightarrow C B$ transition in $\mathrm{ZnTe}: 0$ extracted from the data in

The IB-CB absorption reaches $700 \mathrm{~cm}^{-1}$ at the highest point, being at least over an order of magnitude lower than the absorption for the VB-IB transition. It has to be taken into account that an FTIR spectrometer the sample is illuminated with light of a broadband spectrum, including those wavelengths that produce VB-IB transitions in $\mathrm{ZnTe}: \mathrm{O}$. This illumination is not as high as to produce a situation of large photo-filling of the IB, but can still provide the IB with a sufficient carrier population that allows the measurement of this IB-CB absorption. On the other hand, it is not established yet whether the position of the Fermi level in the practical $\mathrm{ZnTe}: \mathrm{O}$ material is the same than in $\mathrm{ZnTe}$. It has to be noted that the amount of oxygen introduced is huge. Assuming an $\mathrm{O}$ incorporation of $10^{19}$ $\mathrm{cm}^{-3}$, if $1 \%$ of the $\mathrm{O}$ atoms, for example, do not occupy Tesubstitutional sites, there would be $10^{17} \mathrm{~cm}^{-3}$ potential dopants, which may produce complexes that are not necessarily acceptors. In these considerations it should not be forgotten the self-compensation effects that prevent the achievement of a notable n-doping in ZnTe [14].
Finally, it is worth mentioning the IB-CB transition in ZnTe:O shows an abrupt selective character: no absorption is recorded for energies higher than $0.5 \mathrm{eV}$. As explained in the introduction, this is a positive feature from the point of view of IBSC implementation (selectivity of the absorption coefficients), although it will probably need to be accompanied by a blue-shift of the IB-CB gap to render the potential for really high conversion efficiencies. The theoretical results in $[7,12,13]$ indicate that this blue-shift can be produced and by increasing the $\mathrm{O}$ content. In the next section we will discuss quantum efficiency results which show in practice that tendency.

\section{COMPARISON TO QUANTUM EFFICIENCY}

Another way of studying the sub-bandgap absorption of an IB material is to study the QE of IBSCs fabricated with it. The interpretation is not straightforward, since an IBSC requires two sub-bandgap photons to produce one electron-hole pair. In a QE experiment the cell is illuminated with monochromatic light. Therefore, in the ideal case of selectivity of the absorption coefficients, the measurement of sub-bandgap QE is only possible if the promotion of carriers from IB to CB is assisted by thermal carrier escape processes. This has been studied more extensively for the quantum dot based IBSC [15, 16]. In the case of a ZnTe-based IBSC there is the additional difficulty that the $\mathrm{ZnTe}$ cannot be doped n-type. The introduction of a heterojunction is then required for implementing the $\mathrm{n}$ type emitter which also complicates the interpretation of $Q E$ measurements.

We will compare here the $\mathrm{QE}$ of the three devices depicted in Figure 7. They were also grown by MBE, in this case on $n^{+}-G a A s$ substrates. Solar cell devices were fabricated using standard photolithography and metallization ( $\mathrm{Ni} / \mathrm{Au}$ for the $\mathrm{p}$ - and $\mathrm{Ti} / \mathrm{Au}$ for the $\mathrm{n}$-contact), and wet chemical etching to define the mesas. The QE was measured using a grating monochromator (with a set of filters to avoid artifacts caused by the grating secondaries) and a lock-in amplifier

The two samples in Figure 8(a) have the same structure, with the difference that the reference sample SA has a 1 $\mu \mathrm{m}$ absorber of ZnTe and the IBSC SB has a ZnTe:O absorber. In this simple p-on- $n$ structure, the $n^{+}-G a A s$ substrate acts as the base of the pn-junction. Note that, because GaAs has a lower bandgap (1.4 eV) than $\mathrm{ZnTe}$ $(2.3 \mathrm{eV})$, these samples cannot achieve the voltages associated with the ZnTe gap. Sample SC in Figure 8(a) is an IBSC with a more advanced structure where an $n^{+}$$\mathrm{ZnSe}$ base (gap $2.7 \mathrm{eV}$ ) has been inserted between the $\mathrm{ZnTe}: \mathrm{O}$ IB material and the GaAs substrate. Another relevant difference between $S B$ and SC is the amount of oxygen in the IB material: SB was grown with an $O$ flow rate of $1 \mathrm{sccm}$ (the same as sample studied in the absorption measurements) and SC was grown with a lower flow of $0.2 \mathrm{sccm}$. 


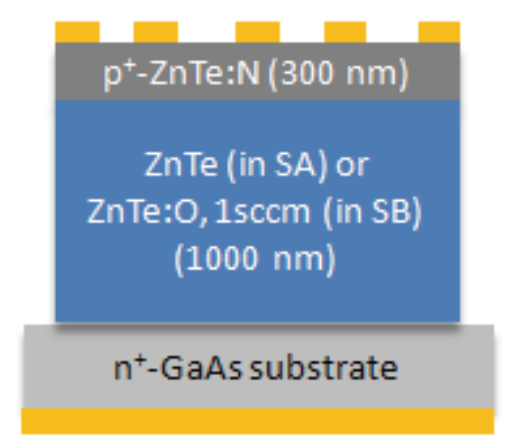

(a) Structure of samples SA (ZnTe reference cell) and SB (ZnTe:O IBSC with high O content). There is no $n^{+}-\mathrm{ZnSe}$ base.

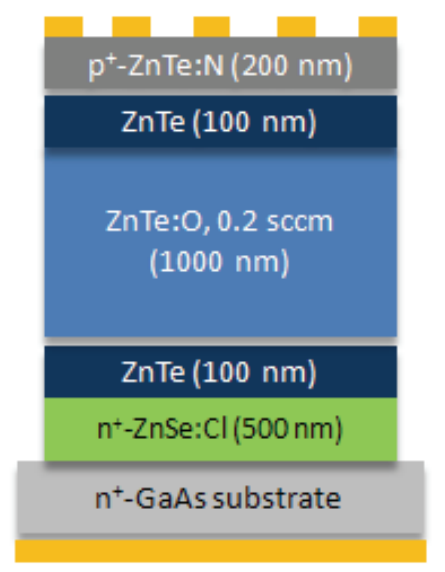

(b) Sample SC: ZnTe:O IBSC

(low $\mathrm{O}$ content, $\mathrm{n}^{+}-\mathrm{ZnSe}$ base)

Figure 7 Structure of the $\mathrm{ZnTe}(: 0)$ solar cell samples.

Figure 8 shows the QE measured on samples SA and SB (upper plot) and sample SC (lower plot). It is interesting to note that all three samples exhibit sub-bandgap photocurrent. In the case of the reference SA, it is easily identified as photogeneration produced at the GaAs substrate. For sample SB there is a clear peak at 1.7-1.8 $\mathrm{eV}$ that can be related to the oxygen, while in SC this peak is less pronounced and has been blue-shifted to a higher energy of about $2 \mathrm{eV}$. This different energies corresponding to differing oxygen content is in agreement with the theoretical predictions and corroborates the possibility of tuning the IB position within the bandgap to a more desirable one for increasing the efficiency of the ZnTe:O based IBSC.

As a last remark, it is worth mentioning that in both IBSC devices, SB and SC, the edge of the sub-bandgap QE coincides with the gap of GaAs. In the case of SC, due to the $500 \mathrm{n}^{+}-\mathrm{ZnSe}$ barrier it seems a priori unlikely that the GaAs substrate can inject holes into the pn-junction.
However, we have observed this GaAs signature in the QE of ZnTe:O IBSC of various structures. Therefore, we suggest that the contribution from the substrate should be considered when studying the photocurrent of this type of structures.
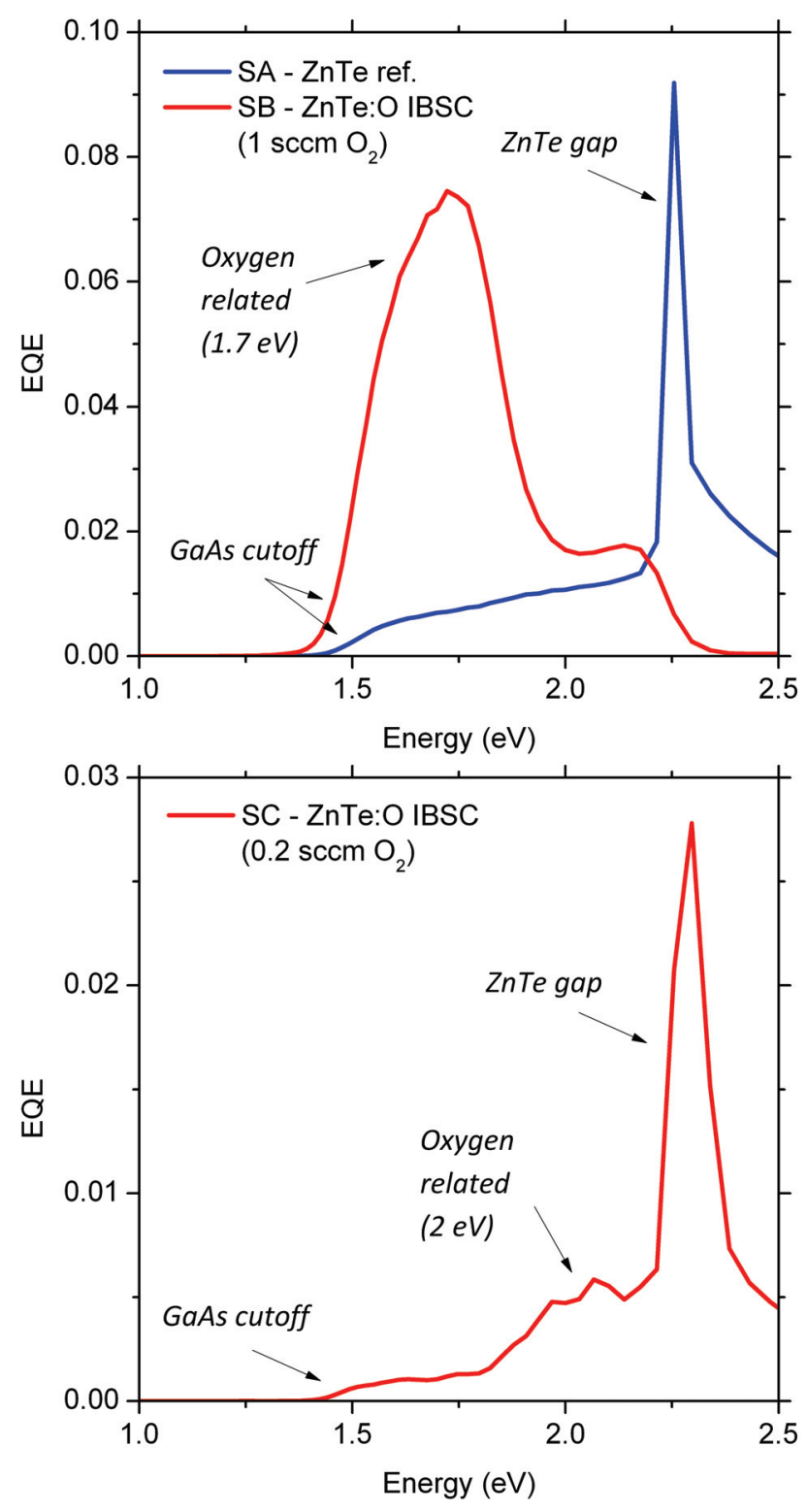

Figure 8 Measured quantum efficiency of the $\mathrm{ZnTe}(: 0)$ solar cells, samples SA and SB (top) and SC (bottom).

\section{CONCLUSIONS}

We have presented the first report to our knowledge of absorption in both sub-bandgap transitions in an IBSC material at room temperature. The material was $\mathrm{ZnTe}: \mathrm{O}$ grown by MBE and, in particular, it was possible to 
measure using an FTIR spectrometer the less energetic IB-CB transition. The extracted absorption coefficient for that transition is $700 \mathrm{~cm}^{-1}$. That low value is not surprising taking into account the natural $\mathrm{p}$-character of the $\mathrm{ZnTe}$ material (the IB is expected to be empty under equilibrium), where determination of the optical cross section (oscillator strength) independent of carrier population would be of great value. The absorption shows a peak at about $0.4 \mathrm{eV}$ and is negligible for energies above $0.5 \mathrm{eV}$. Therefore, it is concluded that the absorption coefficients for the VB-IB transition and IB-CB transition do not overlap in energies but the energy of the IB-CB transition is too low for an efficient IBSC operation. It is important to shift that energy to higher values. We have presented QE characterization of the VB-IB transition in two devices fabricated with $\mathrm{ZnTe}: \mathrm{O}$, showing a redshifting of the VB-IB transition energy with increasing oxygen content.

\section{ACKNOWLEDGEMENTS}

This work has been funded by the bilateral US-Spain Research Programme with Contract C11.0910B.01. The group at UM acknowledges also the National Science Foundation Materials World Network DMR-1006154. EA acknowledges a Juan de la Cierva Post-Doctoral Fellowship (JCl-2011-10639) from the Spanish Ministry of Science. IR and EL acknowledge PhD grants from the Universidad Politécnica de Madrid and the Spanish Ministry of Science (BES-2010-037659), respectively.

\section{REFERENCES}

[1] A. Luque and A. Martí, "Increasing the efficiency of ideal solar cells by photon induced transitions at intermediate levels", Phys. Rev. Lett. 78, pp. 5014-5017, 1997.

[2] A. Luque and A. Martí, "A metallic intermediate band high efficiency solar cell", Prog. Photovolt: Res. Appl. 9, pp. 73-86, 2001.

[3] P. Palacios, I. Aguilera, K. Sanchez, et al., "TransitionMetal-Substituted Indium Thiospinels as Novel IntermediateBand Materials: Prediction and Understanding of Their Electronic Properties", Physical Review Letters 101, pp. 046403-4, 2008.

[4] A. Martí, L. Cuadra, and A. Luque, "Quantum dot intermediate band solar cell", in Conference Record of the 28th IEEE Photovoltaics Specialists Conference, New York, USA, 2000, pp. 940-943.

[5] R. Strandberg and T. W. Reenaas, "Photofilling of intermediate bands", J. of Appl. Phys. 105, p. 124512, 2009.

[6] A. Luque, A. Martí, E. Antolín, and C.Tablero, "Intermediate bands versus levels in non-radiative recombination", Physica B: Condensed Matter 382, pp. 320-327, 2006.

[7] K. M. Yu, W. Walukiewicz, J. Wu, et al., "Diluted II-VI Oxide Semiconductors with Multiple Band Gaps", Phys. Rev. Lett. 91, pp. 246403-4, 2003.

[8] W. Wang, A. S. Lin, and J. D. Phillips, "Intermediate-band photovoltaic solar cell based on ZnTe:O", Appl. Phys. Lett. 95, p. $011103,2009$.
[9] A. S. Lin, W. Wang, and J. D. Phillips, "Model for intermediate band solar cells incorporating carrier transport and recombination", J. Appl. Phys. 105, p. 064512, 2009.

[10] T. Tanaka, K. M. Yu, A. X. Levander, et al., "Demonstration of $\mathrm{ZnTe}_{1-\mathrm{x}} \mathrm{O}_{\mathrm{x}}$ Intermediate Band Solar Cell", Japanese Journal of Applied Physics 50, p. 082304, 2011.

[11] W. Wang, W. Bowen, S. Spanninga, et al., "Optical Characteristics of ZnTeO Thin Films Synthesized by Pulsed Laser Deposition and Molecular Beam Epitaxy", J. Electronic Materials 38, pp. 119-125, 2009.

[12] C. Tablero, "Acceptor and donor ionization energy levels in O-doped ZnTe", Computational Materials Science 49, pp. 368$371,2010$.

[13] C. Tablero, A. Martí, and A. Luque, "Analyses of the intermediate energy levels in ZnTe:O", Appl. Phys. Lett. 96, p. 121104, 2010.

[14] S. B. Zhang, W. Su-Huai, and Z. Alex, "A phenomenological model for systematization and prediction of doping limits in II--VI and I--III--VI[sub 2] compounds", Journal of Applied Physics 83, pp. 3192-3196, 1998.

[15] A. Martí, E. Antolín, C. R. Stanley, et al., "Production of Photocurrent due to Intermediate-to-Conduction-Band Transitions: A Demonstration of a Key Operating Principle of the Intermediate-Band Solar Cell", Physical Review Letters 97, p. 247701(4), 2006.

[16] E. Antolín, A. Martí, C. D. Farmer, et al., "Reducing carrier escape in the InAs/GaAs quantum dot intermediate band solar cell", Journal of Applied Physics 108, pp. 064513 (1-7), 2010. 